\title{
Erbium-doped Yttria-stabilized Zirconia thin layers for photonic applications
}

\author{
(Student paper)
}

\author{
Alicia Ruiz-Caridad ${ }^{1}$, Guillaume Marcaud ${ }^{1}$, Joan Manel Ramirez ${ }^{2}$, Ludovic Largeau ${ }^{1}$, Thomas Maroutian ${ }^{1}$, \\ Sylvia Matzen ${ }^{1}$, Carlos Alonso-Ramos ${ }^{1}$, Guillaume Agnus ${ }^{1}$, Sylvain Guerber ${ }^{1,3}$, Charles Baudot ${ }^{3}$, Frederic \\ Boeuf $^{2}$, Vladyslav Vakarin ${ }^{1}$, Elena Duran-Valdeiglesias ${ }^{1}$, Eric Cassan ${ }^{1}$, Delphine Marris-Morini ${ }^{1}$, Philippe \\ Lecoeur $^{1}$, Laurent Vivien ${ }^{1}$. \\ ${ }^{1}$ Centre de Nanosciences et de Nanotechnologies, CNRS, Univ. Paris-Sud, Université Paris-Saclay, C2N - \\ Palaiseau, 91120 Palaiseau, France \\ ${ }^{2}$ III-V lab, a joint lab from Nokia Bell Labs, Thales and CEA, 1 avenue Augustin Fresnel, 91767 Palaiseau \\ Cedex \\ ${ }^{3}$ TR\&D STMicroelectronics SAS, Crolles, 38920, France \\ e-mail: alicia.ruiz-caridad@c2n.upsaclay.fr
}

\begin{abstract}
Near-infrared (near-IR) integrated photonic devices in silicon based platforms have been studied over the last decades for applications such as on-chip optical communications and sensing. Hybrid integration of functional oxides to search the limits of low power consumption has been a challenge overcome by material engenieering. In this regard, Yttria-Stabilized Zirconia (YSZ) stands as an interesting material for its structural, chemical and optical properties which includes transparency range from the visible to the mid-IR wavelength range. In this regard, we recently demonstrated YSZ waveguides with propagation losses as low as $2 \mathrm{~dB} / \mathrm{cm}$ at a wavelength of $1380 \mathrm{~nm}$ [2]. Based on the encouraging preliminary results, we have recently explored the possibility to introduce active rare-earth dopants into YSZ waveguides to demonstrate on-chip optical amplifiers based on YSZ. In this study, we introduced multilayer approach depositing by pulsed laser deposition (PLD) technique by $\mathrm{Er}^{3+}$ ions, providing outstanding luminescence around $\lambda=1.54 \mu \mathrm{m}$, in correspondence with C-band of telecommunications. Such active layers have been grown onto different platforms, including SiN and sapphire. The optical properties of Er-doped YSZ thin films growth on waveguides under resonant pumping will be discussed in this paper. These results pave the way towards the implementation of new rare-earth-doped functional oxides into hybrid photonic platforms in a customized and versatile manner, adding light amplification functionalities.
\end{abstract}

Keywords: functional oxides, nanophotonics, rare-earth, yttria-stabilized zirconia, optical gain, hybrid photonic platform.

\section{INTRODUCTION}

Latest advances in nanophotonics has triggered the search for lower power consumption of opto-electronic and photonic devices. In this regard, engineering materials has been studied new functional materials to develop miniaturized hybrid photonic integration on silicon based platforms for applications including datacom, telecom, sensing, or quantum optics. In this context, functional oxides have been revealed as very interesting class of materials due to their singular characteristics comprising superconductivity, magnetism, ferroelectricity, catalytic activity, resistive switching, electro-optic effects, piezoelectricity, or optical nonlinearities, among others [1].

Despite the difficulties of hybrid integration on silicon photonic due to lattice mismatch between silicon and other materials, material engineering has found a technique to epitaxially grow high-quality crystalline functional oxide films on silicon keeping the interfaces light scattering to minima [2]. This technique requires a buffer layer for lattice adaptation between silicon and the functional oxide and to avoid interdiffusion between substrate and oxide materials.

One of the more popular oxides in this regarding is yttria-stabilized zirconia (YSZ), extensively used as a buffer layer for functional oxides in applications as a solid electrolyte, gas sensors and fuel cells. YSZ outstanding properties include thermal and chemical stability and hardness and mechanical durability. Moreover, its optics 
optical properties as its high refractive index, large optical band gap that prevents two photon absorption (TPA) in the near and mid-IR; transparency from the ultraviolet to the near-infrared and a good Kerr effect puts this buffer oxide under the scope for photonic integration.

Rare-earths have been used as active materials for photonic devices to enhance light emission in integrated optics to develop new light sources and optical amplifiers. One of the main challenges to confront on rare-earth integration on a host is to maintain the higher level of doping avoiding quenching effects and maintaining the high level of crystallinity of the host. Moreover, only low-phononic materials which avoids non-radiative effects are suitable to host rare-earths. Amplification has been demonstrated in functional oxides as LiNbO3 and gain for oxides as $\mathrm{Al} 2 \mathrm{O} 3$ and $\mathrm{Y} 2 \mathrm{O} 3$, and have been successfully integrated in photonic devices. Y2O3 thin films are good candidates as hosts for rare-earth ions in constructing active waveguides for telecommunication applications operating at $1.55 \mathrm{um}$. Among the rare-earth ions, erbium ion is known to have several transitions in the wavelength range within the near-infrared (near-IR) making this rare-earth a perfect candidate for telecommunication applications. Moreover, it has been demonstrated that erbium ions have good solubility in YSZ.

\section{Experimental procedure}

On a first approach, erbium-doped yttria stabilized-zirconia (Er:YSZ) thin films were deposited by pulsed laser deposition (PLD) on silicon dioxide $\left(\mathrm{SiO}_{2}\right)$ encapsulated silicon nitrate $\left(\mathrm{Si}_{3} \mathrm{~N}_{4}\right)$ waveguides on a silicon platform (Fig.1 (a)) at $800^{\circ} \mathrm{C}$ in an oxygen atmosphere. Laser ablation of two rotating targets of $8 \%$ mol YSZ ceramic target and metallic erbium was carried out by a $\mathrm{KrF}$ excimer laser (248-nm wavelength, 3-J/cm² fluence, and 5$\mathrm{Hz}$ repetition rate).

a)

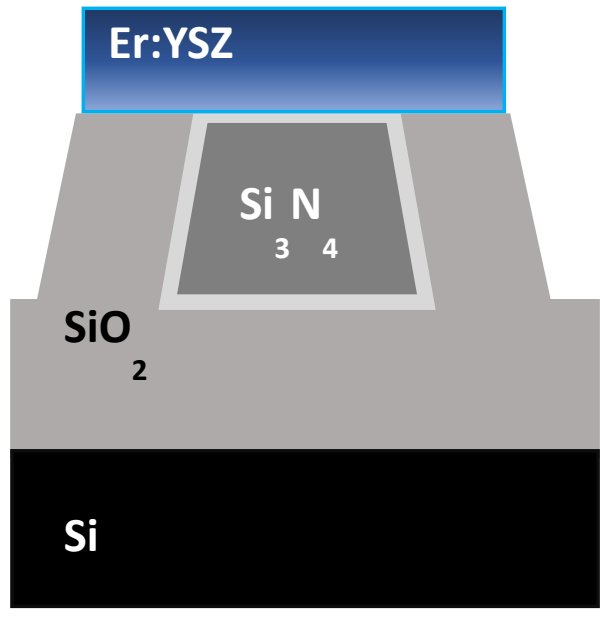

c)

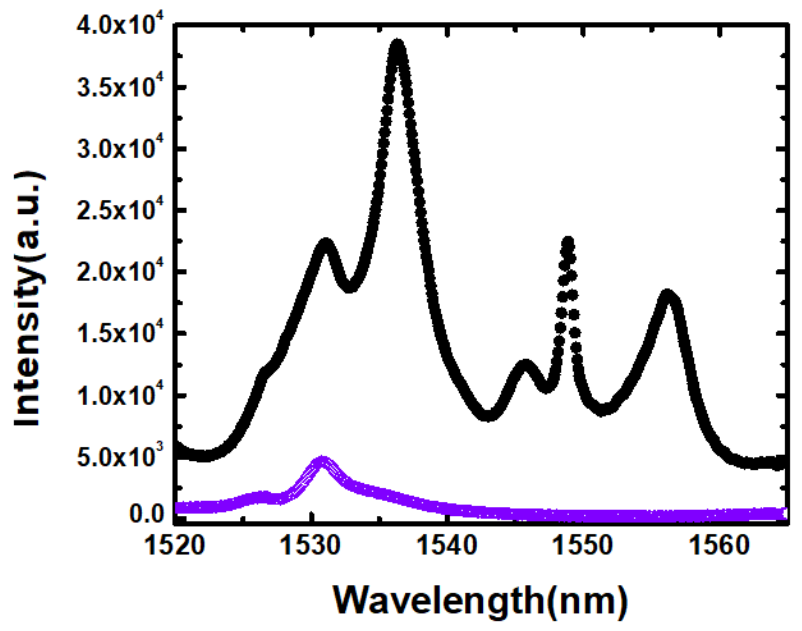

d) 

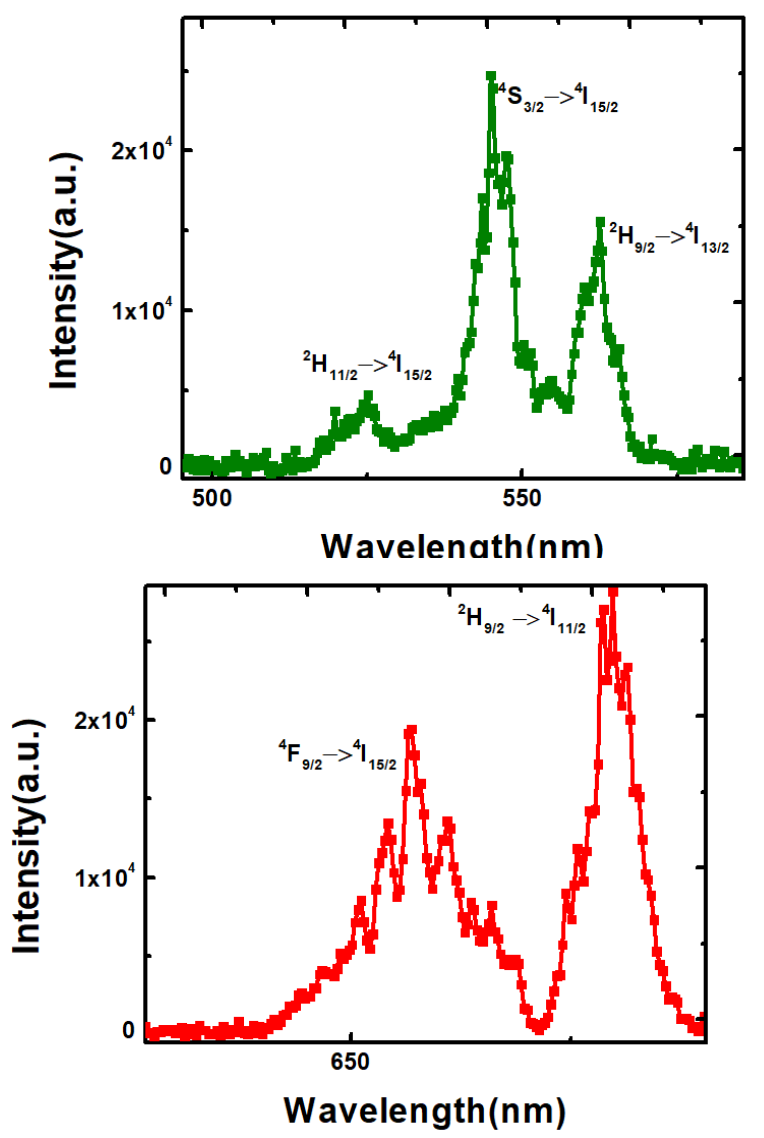

Figure 1. (a) Cross-section of a SiNx waveguide encapsulated by silicon dioxide on a silicon platform. A thin film of Er-YSZ was growth by PLD. (b) PL emission in the near-IR range of Er:YSZ thin film reveals two emissions of different intensities corresponding to the same transition: (black dotted) a locally enhanced signal showing Stark splitting for 1530, 1536 and 1548 nm and (purple dotted) an homogeneous emission with a maximum at $1530 \mathrm{~nm}$. Spectroscopy in the visible range of wavelengths proves erbium up-conversion at different transitions corresponding to the green (c) and red (d) wavelengths.

\subsection{Optical characterization}

In figure 1 (b) we observe two photoluminescence (PL) emission profiles corresponding both to erbium ${ }^{4} \mathbf{I}_{13 / 2} \rightarrow$ ${ }^{4} \mathbf{I}_{\mathbf{1 5 / 2}}$ transition after exciting erbium-doped YSZ thin film at $980 \mathrm{~nm}$ with a continuous wave of an Helium-Neon (He-Ne) laser. PL purple dots signal in figure 1 (b) is homogeneously distributed on the sample and shows a higher intensity at $1530 \mathrm{~nm}$. Moreover, a local emission profile in figure 1(b) (black dots) proves Stark effect of the same transition with enhanced emissions at 1530, 1536 and 1548nm. Stark effect in rare-earth transitions is known to be related to the crystallinity of the sample, out of the scope of our study.

To further optically characterize our sample he have performed visible sprectroscopy by pumping Er:YSZ on $\mathrm{Si}_{3} \mathrm{~N}_{4}$ waveguides at a wavelength of $1480 \mathrm{~nm}$. It has been evidenced strong up-conversion luminescence of erbium ions at 525, 544, 562, 656 and $679 \mathrm{~nm}$ corresponding to ${ }^{2} \mathrm{H}_{11 / 2} \rightarrow{ }^{4} \mathrm{I}_{15 / 2},{ }^{4} \mathrm{~S}_{3 / 2} \rightarrow{ }^{4} \mathrm{I}_{15 / 2},{ }^{2} \mathrm{H}_{9 / 2} \rightarrow{ }^{4} \mathrm{I}_{13 / 2}$, ${ }^{4} \mathrm{~F}_{9 / 2} \rightarrow{ }^{4} \mathrm{I}_{15 / 2}$ and ${ }^{2} \mathrm{H}_{9 / 2} \rightarrow{ }^{4} \mathrm{I}_{11 / 2}$ transitions, respectively [5] . At the shadow of these results, optical gain might be expected.

\section{CONCLUSIONS}

To sum up, high quality crystalline erbium-doped YSZ thin films were epitaxially grown on sapphire substrate and polycrystalline thin films on SiNx waveguides. Optical characterization by means of photoluminescence at 980nm reveal erbium ${ }^{4} \mathbf{I}_{\mathbf{1 3 / 2}} \rightarrow{ }^{4} \mathbf{I}_{\mathbf{1 5 / 2}}$ transition for all the substrates and platforms and it has been noticed a local multiple emission corresponding to Stack effect with enhancement of luminescence. ${ }^{2} \mathrm{H}_{11 / 2} \rightarrow{ }^{4} \mathrm{I}_{15 / 2},{ }^{4} \mathrm{~S}_{3 / 2} \rightarrow{ }^{4} \mathrm{I}_{15 / 2}$ $,{ }^{2} \mathrm{H}_{9 / 2} \rightarrow{ }^{4} \mathrm{I}_{13 / 2},{ }^{4} \mathrm{~F}_{9 / 2} \rightarrow{ }^{4} \mathrm{I}_{15 / 2}$ and ${ }^{2} \mathrm{H}_{9 / 2} \rightarrow{ }^{4} \mathrm{I}_{11 / 2}$ transitions have been measured by visible spectroscopy revealing strong erbium up-conversion when pumping at $1480 \mathrm{~nm}$. 
Future work will focus on the study of gain in Er:YSZ on the hybrid $\mathrm{SiN}_{\mathrm{x}}$ platform at $1.53 \mu \mathrm{m}$ to demonstrate on-chip optical amplifiers at telecom wavelengths.

\section{ACKNOWLEDGEMENTS}

This work has received the funding from the European Research Council (ERC) under the European Union's Horizon 2020 Research and Innovation Program (ERC POPSTAR - grant agreement No 647342).

\section{REFERENCES}

[1] M.W. Madea, et al.: The effect of four-wave mixing in fibres on optical frequency-division multiplexed systems, J. Lightwave Technol., vol. 8. pp. 1402-1408, Sept. 1990.

[2] M. Jaworski, M. Marciniak: Long distance analog CATV link utilizing non-zero dispersion-shifted fiber, in Proc. ICTON 2000, Gdansk, Poland, June 2000, paper TuP5. 